\title{
Is maternal transmission of coronary heart disease risk stronger than paternal transmission?
}

\author{
S Kinra, G Davey Smith, M Okasha, P McCarron, J McEwen
}

See end of article for authors' affiliations

Correspondence to:

Dr Sanjay Kinra, Canynge Hall, Whiteladies Road,

Bristol BS8 2PR, UK;

Sanjay.Kinra@bristol.ac.uk

Accepted

11 December 2002

\begin{abstract}
Objective: To test whether intergenerational transmission of coronary heart disease (CHD) to offspring is greater from the mother than from the father, the association between parental history of CHD and coronary mortality in male offspring was examined.

Design: Prospective cohort study with 43 years of follow up.

Setting: University of Glasgow.

Participants: Male students ( $n=8402$ ) aged 16-30 years when examined in 1948 to 1968 .

Main outcome measure: Fatal CHD.

Main results: Of the 8402 men studied, $615(7.3 \%)$ reported a history of CHD in at least one of the parents: $479(5.8 \%)$ for fathers only, $124(1.6 \%)$ for mothers only, and a further $12(0.2 \%)$ for both their parents. During follow up, $373(4.4 \%)$ men died of CHD. Parental history of disease was associated with fatal CHD and controlling for personal risk factors such as cigarette smoking, body mass index, systolic blood pressure, and father's social class did not attenuate this relation. The fully adjusted hazard ratios were 1.53 (95\% confidence interval (Cl) 1.08 to 2.18), 1.19 (95\% $\mathrm{Cl} 0.61$ to 2.32), and $8.65(95 \% \mathrm{Cl} 2.65$ to 28.31$)$ for father only, mother only, and both parents with $\mathrm{CHD}$, respectively, compared with men whose parents did not have CHD. There was some evidence for interaction between parental histories $(p=0.049)$, with particularly high risk if both parents reported a history of CHD.

Conclusions: This study found no differential transmission of CHD. Paternal history of CHD was at least as important as maternal history. Data from other comparable cohorts provide no consistent evidence of differential transmission. Intergenerational transmission of CHD does not appear to have differential effects between mothers and fathers.
\end{abstract}

$\mathrm{P}$ arental history of coronary heart disease (CHD) has long been recognised as a risk factor for CHD. ${ }^{1}$ Little attention has been paid to the possible differential transmission from mothers or fathers. This has relevance for the "fetal origins" hypothesis in which the association between low birth weight and later CHD is taken to be evidence of fetal programming caused by adverse circumstances in intrauterine life. ${ }^{2}$ Although there is now a substantial body of evidence in support of the association between low birth weight and $\mathrm{CHD}^{3}$ evidence for fetal programming from other lines of inquiry is remarkable by its absence. The questionable validity of low birth weight as a proxy for adverse intrauterine circumstances ${ }^{4}$ makes it all the more important that support for this hypothesis be sought from other types of studies if these associations are to be regarded as causal.

One area of study is parent-offspring disease associations. Maternal factors-for example, cigarette smoking and hypertension - which strongly determine the risk of low birth weight in the offspring, ${ }^{5}$ are also ones that strongly influence the mother's own risk of CHD. ${ }^{6}$ If "fetal programming" occurs, then one would expect to find a greater maternal-offspring than paternal-offspring association for CHD, since modifiable factors influencing paternal CHD risk do not influence the fetal growth rate to the same extent as maternal factors. Greater maternal-offspring transmission of type II diabetes is well established ${ }^{7}$ and has been shown to be associated with intrauterine exposure to diabetes. ${ }^{8}$ Evidence on differential transmission of CHD, however, is mixed. Case control studies that suggest an excess in paternal transmission of CHD have been criticised because of their potential for recall bias.9-11 Cohort studies offer a more reliable study design, but the ones conducted so far have been few and inconclusive. ${ }^{12-18}$ One reason is the lack of history of disease data collected separately for both parents rather than as a combined "parental history of CHD" category in most longstanding cohorts. Availability of such information in an established cohort of students from the University of Glasgow-with over 40 years of follow up for mortality-has allowed us to examine the question of differential transmission of CHD from parents to the male offspring. Studying this question is important not just for testing the fetal origins hypothesis, with attendant policy implications, ${ }^{19}$ but also has value for clinicians in risk assessment and in guiding genetic research.

\section{METHODS}

Details of the Glasgow student cohort are presented elsewhere. ${ }^{20}$ Briefly, between 1948 and 1968, all students registered at the University of Glasgow were invited to attend annual health checks at the Student Health Service. Half of the student population attended at least one examination. At each examination, the physician completed a questionnaire covering sociodemographic and medical history details of the student and his or her family. Clinical measurements included height, weight, and blood pressure. These students have been traced and flagged through the UK's National Health Service Register (NHSCR), which provide copies of death certificates, as deaths occur. The representativeness of the students examined and traced to the university population and the general population has already been reported and discussed elsewhere. ${ }^{20}$

Abbreviations: $\mathrm{BMI}$, body mass index; $\mathrm{Cl}$, confidence interval; $\mathrm{CHD}$, coronary heart disease; ICD, International classification of diseases; NHSCR, National Health Service Register 
Table 1 Parental history and risk factor status by CHD mortality

\begin{tabular}{|c|c|c|c|}
\hline & \multicolumn{2}{|c|}{ Death from CHD } & \multirow[b]{2}{*}{$\mathrm{p}$ Value } \\
\hline & $\begin{array}{l}\text { Yes } \\
(n=373)\end{array}$ & $\begin{array}{l}\text { No } \\
(n=8029)\end{array}$ & \\
\hline \multicolumn{4}{|l|}{ Parental history of CHD \% (n) } \\
\hline Neither parent & $87.4(326)$ & $92.9(7461)$ & $<0.001$ \\
\hline Either parent & $12.6(47)$ & $7.1(568)$ & $<0.001$ \\
\hline Father only & $9.7(35)$ & $5.6(444)$ & 0.001 \\
\hline Mother only & $2.7(9)$ & 1.5 (115) & 0.092 \\
\hline Both & $0.9(3)$ & 0.1 (9) & $<0.001$ \\
\hline \multicolumn{4}{|l|}{ Risk factor status } \\
\hline Age (years) & $22.2(0.2)$ & 20.5 (0) & $<0.001$ \\
\hline \multicolumn{4}{|c|}{ Number of cigarettes per day $\%$ (n) } \\
\hline None & $50.4(188)$ & $67.2(5395)$ & \\
\hline $1-10$ & $30.6(114)$ & 24.8 (1988) & \\
\hline $11-20$ & $18.0(67)$ & $7.6(613)$ & \\
\hline Greater than 20 & $1.1(4)$ & $0.4(33)$ & $<0.001$ \\
\hline \multicolumn{4}{|c|}{ Father's social class \% (n) } \\
\hline I & $13.7(51)$ & $20.3(1632)$ & \\
\hline ॥ & 37.3 (139) & $35.8(2875)$ & \\
\hline III & 40.0 (149) & 36.6 (2938) & \\
\hline IV & $6.4(24)$ & $5.8(469)$ & \\
\hline $\mathrm{V}$ & $2.7(10)$ & 1.4 (115) & 0.011 \\
\hline Height $(\mathrm{cm})$ & $174.0(0.3)$ & $174.8(0.1)$ & 0.017 \\
\hline Body mass index $\left(\mathrm{kg} / \mathrm{m}^{2}\right)$ & $21.9(0.1)$ & $21.6(0)$ & 0.010 \\
\hline Systolic BP (mm Hg) & $134.7(0.7)$ & $130.8(0.2)$ & $<0.001$ \\
\hline
\end{tabular}

Numbers are mean (SE) unless stated otherwise.

$\mathrm{BP}$, blood pressure; CHD, coronary heart disease.

\section{Main exposure and outcome}

A positive parental history of CHD was recorded if the student reported a history of angina or of fatal or non-fatal myocardial infarction in either parent; no distinction was made between these outcomes. The main outcome was death from CHD. Cause of death was determined from the death certificate and those participants for whom the cause of death included the International classification of diseases (ICD) codes 410-414 (ICD 9th revision) and I20-I25 (ICD 10th revision) contributed to the outcome.

\section{Follow up period and exclusion criteria}

The follow up period extended from the date of the first examination to 30 November 2001 or the date of death, whichever came earlier. Where the participants were lost to follow up by leaving the UK or entering the armed forces, the last available date of exit was used as the censoring date. Those for whom the last date of exit was not available were excluded from the analyses, as were those with missing date of birth or pre-existing CHD. Although the original cohort had older as well as female students, they were fewer in number and either were spread over a wide age range at examination (older students) or provided too few deaths for analyses (female students). As a result, the present analyses has been limited to male students aged 30 years and under at the time of first examination.

\section{Literature review}

Other comparable cohort studies were identified by systematically searching Medline databases for articles published in any language before February 2001 that reported associations between parental history of disease and offspring CHD. The search strategy, mapped to the medical subject headings, was as follows: ["coronary disease" or "myocardial infarction"] and [ "family health" or "parental history (text word)" or "maternal and paternal history (text word)"] and ["cohort studies"]. Additional studies were identified by hand searching cited references. Data were abstracted for male subjects only.

\section{Statistical methods}

CHD mortality was modelled using Cox regression, with age as the time dependent variable. Parental history of CHD was examined in three categories: paternal history of CHD only, maternal history of CHD only, and both parents with a history of CHD. Hazard ratios were calculated for each of these categories and compared with the baseline category of neither parent having a history of CHD. Potential confounders included in the adjusted models were cigarette smoking (none, 1-10, 11-20, > 20), father's social class (registrar general's classification, I-V), height $(\mathrm{cm})$, systolic blood pressure $(\mathrm{mm} \mathrm{Hg})$, and body mass index (BMI) $\left(\mathrm{kg} / \mathrm{m}^{2}\right)$. BMI was calculated from the weight $(\mathrm{kg})$ and height measurements using Quetelet's index: weight $(\mathrm{kg})$ divided by height $(\mathrm{m})$ squared. Apart from age, all models were adjusted for quintiles of year of birth to account for a possible cohort effect. Interaction terms were introduced between parental history and selected risk factors (and among them) to look for effect modification. The models were tested for proportionality of hazard assumptions. Results from other comparable cohorts identified from the literature were tested for heterogeneity. For studies where the risk estimates or their confidence intervals were not presented in the paper but raw data were available, appropriate calculations were done. ${ }^{14}{ }^{15}$ All analyses were carried out using Stata (version 7.0, Stata Corporation, College Station, Texas, USA).

\section{RESULTS}

Of the 11756 male students who originally attended the student health service, 9930 (85\%) have been traced through the NHSCR. Of these, 292 men were excluded because of unknown date of last exit and a further 381 by virtue of being over age 30 . Those without date of birth $(n=2)$ and those with existing CHD (one student had a history of angina) were also excluded. The remaining 9257 men were available for analyses; information on all the potential confounders was complete for 8402 . The results did not differ materially on exclusion of men with incomplete information. Data presented below are therefore for the 8402 men among whom examination of potential confounding was possible.

Of the 8402 men, $615(7.3 \%)$ reported a history of CHD in at least one parent: $479(5.8 \%)$ for fathers only, 124 (1.6\%) for mothers only, and a further $12(0.2 \%)$ for both their parents.

Table 2 Relation between parental history of CHD and fatal CHD in the male offspring

\begin{tabular}{|c|c|c|c|c|}
\hline $\begin{array}{l}\text { Parental history } \\
\text { of CHD }\end{array}$ & $\begin{array}{l}\text { Number (\%) } \\
\text { deaths }\end{array}$ & $\begin{array}{l}\text { Hazard ratio }(95 \% \mathrm{Cl} \text { ) } \\
\text { (unadjusted) }\end{array}$ & $\begin{array}{l}\text { Hazard ratio }(95 \% \mathrm{CI}) \\
\text { (adjusted for year of birth) }\end{array}$ & $\begin{array}{l}\text { Hazard ratio (95\% } \\
\text { CI) (fully adjusted)* }\end{array}$ \\
\hline $\mathrm{Nei}$ & $326(87.4)$ & 1 & 1 & 1 \\
\hline Either & $47(12.6)$ & 1.60 (1.18 to 2.18$)$ & 1.53 (1.13 to 2.09 ) & $1.53(1.13$ \\
\hline Father only & 35 (9.7) & $1.59(1.12$ to 2.26$)$ & 1.53 (1.08 to 2.17 ) & 1.53 (1.08 to 2.18 ) \\
\hline Mother only & $9(2.7)$ & $1.26(0.65$ to 2.45$)$ & 1.20 (0.62 to 2.33$)$ & $1.19(0.61$ to 2.32$)$ \\
\hline Both parents & $3(0.9)$ & 10.08 (3.23 to 31.46$)$ & 9.88 (3.16 to 30.89 ) & 8.65 (2.65 to 28.31$)$ \\
\hline
\end{tabular}

*Adjusted for year of birth quintile, cigarette smoking, father's social class, height, body mass index, and systolic BP.

†Reference category.

$\mathrm{Cl}$, confidence interval. 


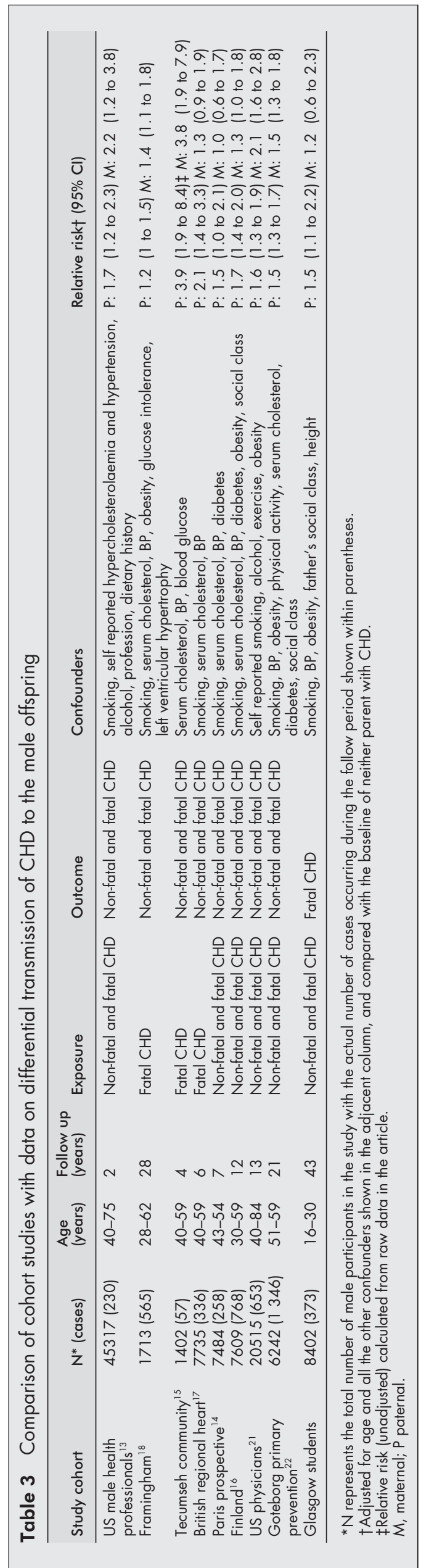

The mean age at examination was 21 years (range 16-30 years). During the subsequent 43 years of follow up, 1002 (11.9\%) men died; 373 (4.4\%) of the deaths were caused by CHD. The significant risk factors for CHD related mortality in this cohort were age, cigarette smoking, father's social class, height, BMI, and systolic blood pressure (table 1). Parental history of CHD was associated with death from CHD. This relation was not attenuated by controlling for other risk factors; fully adjusted hazard ratios were 1.53 (95\% confidence interval (CI) 1.08 to 2.18 ), 1.19 (95\% CI 0.61 to 2.32 ), and 8.65 (95\% CI 2.65 to 28.31 ) for father only, mother only, and both parents with CHD, respectively, compared with the baseline category of neither parent having CHD (table 2). There was some evidence in favour of multiplicative interaction between parental histories $\left(\mathrm{p}_{\text {interaction }}=0.049\right)$.

Eight comparable cohort studies with data on male participants were identified from the literature. ${ }^{13-182122}$ Table 3 summarises their study characteristics and those of the present study. One cohort has since been re-examined but the relative risks presented in the more recent study were not controlled for confounding factors (the unadjusted results do not show differential transmission $)^{23}$; hence, earlier results are included in table $3 .{ }^{14}$ Five of the nine studies (including the current data) have found the central estimates to be in favour of paternal transmission, but there is considerable overlap between the confidence intervals. Significant between study heterogeneity $(p<0.001)$ precluded a quantitative synthesis of results.

\section{DISCUSSION}

These results in a cohort of male students with over 40 years of follow up showed that a paternal history of CHD was positively associated with death due to CHD, whereas the association with maternal history was relatively weaker and did not reach conventional levels of significance. The relative risk for a man with both parents having CHD (as compared with neither parent having CHD) appeared to be greater than that which would be expected simply by multiplying the relative risk from each parent; however, the number of cases in this category was quite small. The overlapping confidence intervals in this study do not allow any firm conclusions about differential transmission, but evidence from other comparable cohorts suggests that it is unlikely.

\section{Strengths and limitations}

The main strengths of the present study are its large sample size and a long duration of follow up with relatively limited loss of participants. Its greatest strength, however, lies in the early age at which the cohort was established. Knowledge and reporting of family history and risk behaviour are highly susceptible to the experience of disease outcome in the subject. Cohort studies serve their purpose best when exposure information is collected at the baseline. This cohort was aged 16-30 years at the time and only one subject had experienced the outcome (angina).

There are two main limitations to this study: the early age at measurement and completeness of risk factor data, and the possibility of selection bias. Risk factor information was collected at one point in time in young adulthood and may have changed over time; data on some other established risk factors such as serum cholesterol were not available. Other studies have found the risk factor data in this cohort to be highly predictive of future cardiovascular disease and CHD ${ }^{24-26}$ Moreover, studies that controlled for risk factors from later life or from blood investigations did not report any notable change in the direction or strength of the observed associations. ${ }^{16-18}$ Selection bias is possible, as only half of the students who were invited to take part in the health screening did so; they may have been more health conscious or had a family history of disease. Cohort members and nonparticipants have already been shown to be comparable in 
their sociodemographic characteristics, ${ }^{20}$ and the prevalence rates for family history of disease and disease outcome are comparable with those of other cohorts. Despite this, selection bias remains a possibility and its contribution to the results is impossible to estimate; however, it would be expected to influence the association with both parents similarly, leaving the mutual relation unaltered. A remaining issue is misclassification arising out of the use of offspring reported family history as a measure of exposure (disease in the parent). This measure has been used by other studies and allows direct comparison of the results. Although the validity of this measure was not examined in the present study, other studies that have looked at this have found it to be moderate in sensitivity and high in specificity. ${ }^{17}{ }^{27}$ This would imply that the true estimates for the associations with family history may be higher than those estimated in this study.

\section{Comparison with previous work}

The magnitude of the hazard estimates derived from various studies were similar apart from one study-Tecumseh community, ${ }^{15}$ which also had wide confidence intervals (only 57 cases). Five of the nine studies (including current data) have found the central estimates to be in favour of paternal transmission, ${ }^{14-18}$ but the overlapping confidence intervals (between and among studies) prevent any firm conclusions being drawn. The differences between studies, apart from sampling variation, can also be attributed to the differences in age of the participants and the severity of the disease arising from differing definitions for exposure and outcome. Confounding factors measured in these studies also vary; however, they are unlikely to be a source of great variation-there was little actual difference between the crude and adjusted hazard ratios in the studies. The majority of the studies controlled for the two important risk factors: smoking and hypertension.

\section{Biological plausibility}

The a priori mechanism under study was the fetal origins hypothesis, which proposes in utero programming of chronic diseases caused by fetal malnourishment. ${ }^{2}$ Sharing of common risk factors between $\mathrm{CHD}^{6}$ and low offspring birth weight, ${ }^{5}$ or the greater maternal than paternal heritability of birth weight, ${ }^{28}$ which in turn may be determined genetically or by the fetal environment, would result in stronger maternal-offspring associations for $\mathrm{CHD}_{1}{ }^{29}$ but this was not the case. There are other possible mechanisms by which parental history of a chronic disease such as CHD may be transmitted differentially to the offspring. These differences can arise from differences in genetic susceptibility per se or from differential transmission of risk factors. ${ }^{30}$ Differential transmission of risk factors in turn may arise because of behavioural reasons (selective adoption of risk behaviour depending on the sex of the parent and the offspring) or genetic susceptibility to risk factors. ${ }^{31}$ Differential genetic transmission may conceivably be influenced by the $\mathrm{X}$ chromosome or mitochondrial DNA, or arise out of the process of genomic imprinting. So far, however, there is little evidence to support any of these in the aetiology of CHD.

\section{Implications of the study}

Apart from shedding light on the debate surrounding the fetal origins hypothesis, which in itself has significant resource and health policy implications, ${ }^{19}$ a differential transmission of CHD, if true, has other clinical and research implications. Taking a bedside family history is already an important tool for the clinician and its value in offering prognosis and risk behaviour advice may be enhanced, although the ability of such advice to alter behaviour remains controversial. In epidemiological research and clinical trials, such knowledge may improve risk stratification and limit residual confounding or even guide genetic research as in the case of diabetes mellitus. ${ }^{7}$ Despite the relevance, most of the studies so far have been unable to provide conclusive evidence, possibly because of lack of sufficient number of cases. We have summarised and highlighted the limitations of the existing evidence from prospective studies involving men. A more formal systematic review on the subject, which the authors are currently undertaking, may help to clarify the situation further.

\section{Conclusions}

This study in a cohort of male students with over 40 years of follow up did not find a differential transmission of CHD. Paternal history of CHD was at least as important as maternal history of CHD. Although the overlapping confidence intervals here make firm conclusions difficult, evidence from other comparable cohorts suggests that a significant differential transmission is unlikely. If fetal programming is indeed a dominant mechanism in the aetiology of CHD, then this study has failed to substantiate that hypothesis by showing an excess in maternal transmission. Although the fetal origins hypothesis is now supported by a reasonable level of "consistency of association" between low birth weight and CHD, "coherence of evidence" from different types of study, another very important criterion for causality, remains elusive.

\section{ACKNOWLEDGEMENTS}

The authors acknowledge the financial support of the Stroke Association, Chest Heart and Stroke, Scotland, NHS R\&D CVD Programme, and the World Cancer Research Fund.

\section{Authors' affiliations}

S Kinra, G Davey Smith, M Okasha, Department of Social Medicine, University of Bristol, Bristol, UK

P McCarron, Department of Epidemiology \& Public Health, N Ireland Cancer Registry, The Queen's University of Belfast, Belfast, UK J McEwen, Department of Public Health, University of Glasgow, Glasgow, UK

\section{REFERENCES}

1 Friedlander $Y$. Familial clustering of coronary heart disease: a review of the significance and role as a risk factor for the disease. In: Genetic factors in coronary heart disease. Dordrecht: Kluwer Academic Publishers, 1994

2 Barker DJP. Fetal origins of coronary heart disease. BM 1995;311:171-4.

3 Godfrey KM, Barker DJP. Fetal nutrition and adult disease. Am J Clin Nutr 2000;71 (suppl): 1344S-52S.

4 Wilcox AJ. On the importance - and the unimportance - of birthweight. Int J Epidemiol 2001;30:1233-41.

5 Kramer MS. Determinants of low birthweight: methodological assessment and meta-analysis. Bull World Health Organ 1987:65:663-737.

6 Stamler J. Established major coronary risk factors. In: Marmot M, Elliott $\mathrm{P}$, eds. Coronary heart disease epidemiology: from aetiology to public health. Oxford: Oxford University Press, 1992:35-66.

7 Cox NJ. Maternal component in NIDDM transmission. How large an effect? Diabetes 1994;43:166-8.

8 Dabelea D, Hanson RL, Lindsay RS, et al. Intrauterine exposure to diabetes conveys risks for type 2 diabetes and obesity: a study of discordant sibships. Diabetes 2000;49:2208-211.

9 Thordarson O, Fridriksson S. Aggregation of deaths from ischaemic heart disease among first and second degree relatives of 108 males and 42 females with myocardial infarction. Acta Med Scand 1979;205:493-500.

10 Rissanen AM, Nikkila EA. Identification of the high-risk groups in familial coronary heart disease. Atherosclerosis 1984;53:37-46.

11 Hamsten A, De Faire U. Risk factors for coronary artery disease in families of young men with myocardial infarction. Am J Cardiol 1987;59:14-9.

12 Colditz GA, Stampfer M, Willett WC, et al. A prospective study of parental history of myocardial infarction and coronary heart disease in women. Am J Epidemiol 1986;123:48-58.

13 Colditz GA, Rimm EB, Giovannucci E, et al. A prospective study of parental history of myocardial infarction and coronary artery disease in men. Am J Cardiol 1991;67:933-8.

14 Cambien F, Richard J-L, Ducimetiere P. [Familial history of coronary heart diseases and high blood pressure in relation to the prevalence of risk factors, and the incidence of coronary heart diseases: The Paris prospective study]. Rev Epidemiol Sante Publique 1980;28:21-37.

15 Deutscher S, Ostrander LD, Epstein FH. Familial factors in premature coronary heart disease-a preliminary report from the Tecumseh Community Health Study. Am J Epidemiol 1970;91:233-7. 
16 Jousilahti P, Puska P, Vartiainen E, et al. Parental history of premature coronary heart disease: an independent risk factor of myocardial infarction. J Clin Epidemiol 1996;49:497-503.

17 Phillips AN, Shaper AG, Pocock SJ, et al. Parental death from heart disease and the risk of heart attack. Eur Heart J 1988;9:243-51.

18 Schildkraut JM, Myers RH, Cupples LA, et al. Coronary risk associated with age and sex of parental heart disease in the Framingham Study. Am J Cardiol 1989;64:555-9.

19 Barker DJP. Fetal programming and public health. In: O'Brien PMS, Wheeler T, Barker DJP, eds. Fetal programming: influences on development and disease in later life. London: RCOG Press, 1999:3-11.

20 McCarron P, Davey Smith G, Okasha M, et al. Life course exposure and later disease: a follow-up study based on medical examinations carried out in Glasgow University (1948-68). Public Health 1999.113:265-71.

21 Sesso HD, Lee IM, Gaziano JM, et al. Maternal and paternal history of myocardial infarction and risk of cardiovascular disease in men and women. Circulation 2001;104:393-8.

22 Rosengren A, Thelle D, Wilhelmsen L. Parental age and coronary disease in the general male population. J Intern Med 2002;251:258-67.

23 Jouven X, Desnos M, Guerot C, et al. Predicting sudden death in the population: the Paris prospective study I. Circulation 1999;99:1978-83.
24 McCarron P, Davey Smith G, Okasha M, et al. Smoking in adolescence and young adulthood and mortality in later life: prospective observational study. J Epidemiol Community Health 2000;55:334-5.

25 McCarron P, Davey Smith G, Okasha $M$, et al. Blood pressure in young adulthood and mortality from cardiovascular disease. Lancet 2000;355:1430-1.

26 Davey Smith G, McCarron P, Okasha M, et al. Social circumstances in childhood and cardiovascular disease mortality: prospective observational study of Glasgow University students. J Epidemiol Community Health 2001;55:340-1.

27 Kee F, Tiret L, Robo JY, et al. Reliability of reported family history of myocardial infarction. BM 1993;307:1528-30.

28 Magnus P, Gjessing HK, Skrondal A, et al. Paternal contribution to birth weight. J Epidemiol Community Health 2001;55:873-7.

29 Davey Smith G. Genetic risk factors in mothers and offspring. Lancet 2001;358:1268.

30 Sankaranarayanan K, Chakraborty R, Boerwinkle EA. lonizing radiation and genetic risks. VI. Chronic multifactorial diseases: a review radiation and genetic risks. essential hypertension and diabetes mellitus. Mutat Res 1999;436:21-57

31 Perusse L, Leblanc C, Tremblay A, et al. Familial aggregation in physical fitness, coronary heart disease risk factors, and pulmonary function measurements. Prev Med 1987; 16:607-15.

\section{IMAGES IN CARDIOLOGY}

\section{Electrocardiographic gated multislice computed tomography of the thoracic aorta}

$\mathrm{A}^{\mathrm{s}}$ 72 year old man attended for follow up computed tomography (CT) of a chronic type A aortic dissection, three years on from his original presentation. The contrast enhanced multislice CT was acquired using retrospective ECG gating. Subsequent reconstruction of the CT images was from a diastolic "reconstruction window" centred at $75 \%$ of the RR interval. This mode of acquisition provided images of the thoracic aorta free of motion artefact. The chronic dissection flap could clearly be seen arising in the proximal, aneurysmal ascending aorta (panel A, axial CT section), extending to the head and neck vessels (panel B, sagittal CT section), and progressing distally to the descending aorta (panel C, three dimensional reconstruction in sagittal section). Advanced post-processing using volume rendering techniques demonstrated the dissection (panel C) and the aneurysm (panel D) in three dimension.

Aortic motion artefact is the most common cause of false positive detection of dissection of the ascending aorta. Motion artefact can be reduced by lowering the patient's heart rate but also by the use of ECG gating in the scan acquisition. Selection of the diastolic "reconstruction window" with the best image quality can be predicted according to the patient's heart rate. In this case the heart rate was 65 beats per minute and the "reconstruction window" selected was centred at $75 \%$ of the RR interval.

\section{G J Morgan-Hughes}

C A Roobottom

A J Marshal
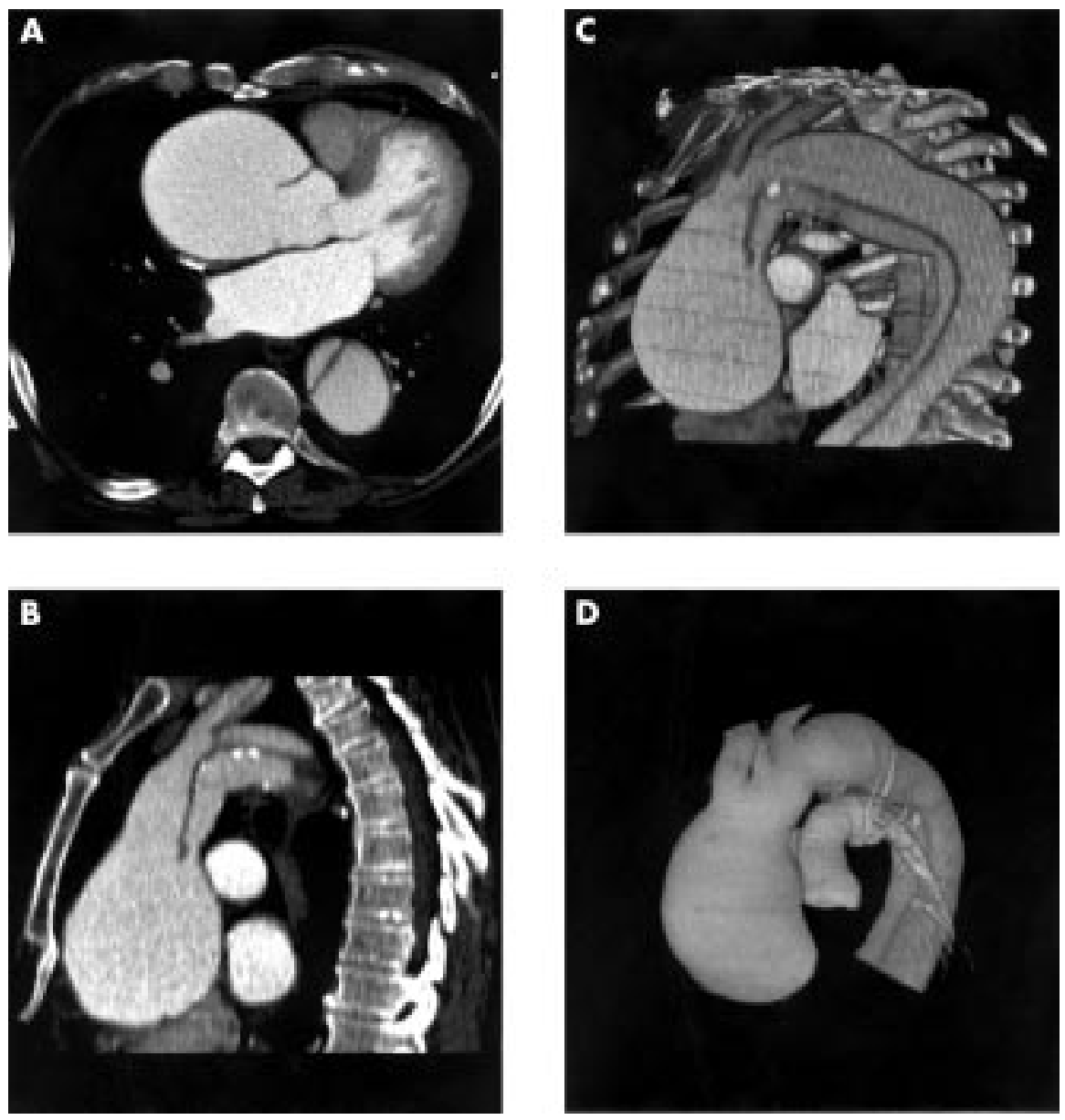